\title{
Knockdown of TRIM44 inhibits the progression of human epithelial ovarian cancer via the FOXM1- EZH2 signaling pathway
}

\section{Fanling Meng}

Harbin Medical University Cancer Hospital

Jing Ding

Harbin Medical University Cancer Hospital

\section{Xihai Chen}

Harbin Medical University Cancer Hospital

\section{Lin Sui}

Harbin Medical University Cancer Hospital

\section{Yuanlong Hu}

The fifth Hospital of Harbin

\section{Shuang Liu}

Harbin Medical University Cancer Hospital

\section{Guangyue Shi}

Harbin Medical University Cancer Hospital

\section{Yunlong He}

Harbin Medical University Cancer Hospital

\section{Xin Ning}

Harbin Medical University Cancer Hosptial

Jiaqi Zhu

Harbin Medical University Cancer Hospital

\section{Ruixue Zhao}

Harbin Medical University Cancer Hospital

\section{Jing Ma}

Harbin Medical University Cancer Hospital

\section{Sujing Ren}

Harbin Medical University Cancer Hospital

\section{Rong Ma ( $\nabla$ dr_marong2017@126.com )}

Harbin Medical University Cancer Hospital https://orcid.org/0000-0002-8392-5580

\section{Ning Huang}

Harbin Medical University Cancer Hospital 
Primary research

Keywords: TRIM44, Ovarian cancer, Invasion, Metastasis

Posted Date: April 15th, 2021

DOI: https://doi.org/10.21203/rs.3.rs-287714/v1

License: (c) (i) This work is licensed under a Creative Commons Attribution 4.0 International License. Read Full License 


\section{Abstract}

Background. Tripartite motif-containing protein 44 (TRIM44) was recently identified as a novel oncogene that is overexpressed in several types of human cancers. However, the biological functions of TRIM44 in epithelial ovarian cancer (EOC) remain unclear. Here, we aimed to investigate the role of TRIM44 in EOC and its clinical implications.

Methods. The expression of TRIM44 in different ovarian cancer cell lines were detected by western blot. TRIM44 was knocked down by shRNA transfection. The in vitro proliferation, invasion, migration and apoptosis of ovarian cancer cells were detected by CCK8, colony formation assay, transwell filters, tube formation assay and flow cytometry analysis, respectively. The growth ability of xenograft tumors in vivo was examined by a nude mouse metastatic tumor model. Finally, we carried out gene chip analysis and IPA to analyze the potential gene network.

Results. High expression of TRIM44 was observed in EOC tissues and cell lines. Knockdown of TRIM44 expression substantially suppressed the proliferation, migration, invasion and colony-forming ability of EOC cells in vitro and attenuated tumor growth in vivo. Mechanistic studies showed that silencing TRIM44 dramatically down regulated the expression of FOXM1, EZH2, CCNE2, CCND3 and BIRC5 in EOC cells, at least in part through inactivation of the FOXM1-EZH2 signaling pathway.

Conclusion. Collectively, these data suggest that TRIM44 downregulation inhibits the progression of EOC cells through the suppression of the FOXM1-EZH2 signaling pathway. These results provide novel insight into the role of TRIM44 in tumorigenesis and suggest it could be a potential therapeutic target of ovarian carcinoma.

\section{Introduction}

Ovarian cancer is one of the most common human malignancies in women, resulting in an estimated 239,000 new diagnoses worldwide each year[1]. Epithelial ovarian cancer (EOC) is the most common histological type of ovarian cancer, accounting for $80 \%$ to $90 \%$ of malignancies. Approximately $70 \%$ of EOC patients are diagnosed with advanced stage disease, which has the highest mortality rate among all female malignancies. In China, there were approximately 52,100 new cases and 22,500 deaths caused by EOC in 2015[2].The prognosis for patients suffering from EOC remains poor because of the limited therapeutic strategies and late diagnosis. Despite improvements in treatments for ovarian cancer, including debunking surgery, platinum-based chemotherapy and targeted therapy, the 5-year survival rate of advanced ovarian cancer is only approximately $30 \%[3]$.Therefore, investigating predicative biomarkers and understanding the molecular mechanism underlying EOC development are important for developing effective therapeutic targets.

TRIM44, a member of the tripartite motif-containing protein (TRIM) family, has been documented to be overexpressed in various types of cancer, such as osteosarcoma[4], lung cancer[5], breast cancer[6], papillary thyroid cancer[7], testicular germ cell tumor[8]and prostate cancer[9]. In addition, high 
expression of TRIM44 is significantly associated with a poor prognosis in malignant tumors[10, 11]. Our previous study also demonstrated that high expression of TRIM44 protein in ovarian tissues is significantly correlated with clinicopathological factors and poor prognosis[12]. However, the expression of TRIM44 in ovarian cell lines and its mRNA level in ovarian tissues still need to be explored. In addition, the biological functions and carcinogenic mechanisms of TRIM44 in epithelial ovarian cancer (EOC) remain unclear.

Here, we report that the TRIM44 protein and mRNA levels are frequently increased in epithelial ovarian cancer clinical tissues and cell lines. Using lentivirus-driven shRNA knockdown, cell proliferation, migration, invasion, colony formation, and apoptosis analyses and in vivo animal experiments, we demonstrated that TRIM44 promotes EOC cell proliferation, migration, invasion, and colony formation and xenograft tumor growth. Furthermore, we showed that TRIM44 down regulation inhibits the carcinogenesis of EOC cells in part through the suppression of the FOXM1-EZH2 signaling pathway. Our data suggest that TRIM44 may be a potential therapeutic target for the treatment of ovarian cancer.

\section{Materials And Methods}

\section{Patient samples}

Five fresh EOC samples and 3 normal ovarian tissue samples were collected from Harbin Medical University Cancer Hospital (Harbin, China). All patients underwent maximal cytoreduction followed by platinum-based combination chemotherapy. None of the patients received chemotherapy, radiotherapy or immunotherapy before surgery. This study's protocol was approved by the Ethics Committee of Harbin Medical University Cancer Hospital.

\section{Cell lines and cell culture}

The human EOC cell lines CAOV3, OVCAR3, A2780, HO-8910PM, and SKOV3 were obtained from the Cell Resource Center of the Chinese Academy of Sciences (Shanghai, China). The cells were cultured in RPMI1640 medium (Gibco BRL, Grand Island, NY, USA) containing 10\% fetal calf serum (FBS, HyClone, Australia). All cells were maintained in a humidified atmosphere containing $5 \% \mathrm{CO}_{2}$ at $37^{\circ} \mathrm{C}$.

\section{Lentiviral vector encoding shRNA targeting TRIM44}

ShRNA was designed by Ambion Company's online design tool. The lentiviral vector encoding TRIM44 shRNA was synthesized and packaged by Ruisai Biotechnology Co., Ltd. (Shanghai, China).The lentiviral vector carrying three types of TRIM44-shRNA was used to screen for maximum silencing efficiency. We electrically transfected the TRIM44-shRNA vectors into SKOV3 and A2780 cells to construct a lentiviral vector encoding shRNA targeting TRIM44, and the empty vector was used as the control. Short hairpin oligonucleotides (shTRIM44: 5'-

TGCTGTGAGTCATCAACCTATCCATGGTTTTGGCCACTGACTGACCATGGATATTGATGACTCA-3' and shCon: 5'-CCTGTGAGTCATCAATATCCATGGTCAGTCAGTGGCCAAAACCATGGATAGGTTGATGACTCAC -3') 
were synthesized and cloned into the lentivirus-based vector pLenti6.3-MCS/V5 DEST (Thermo Electron Corporation, Waltham, MA, USA). Lentivirus production was followed as a standard protocol by transfecting 293T cells with the recombinant shRNA vector, together with psPAX2 and pVSVG(Thermo Electron Corporation, Waltham, MA, USA).

\section{Lentivirus infection}

SKOV3 and A2780 cells were cultured in a six-well plate, and lentiviral vectors with theTRIM44 shRNA sequence or NC shRNA sequence were added at a multiplicity of infection (MOI) of 100to the plate when the cells reached $70 \%$ confluency. The medium was replaced after $48 \mathrm{~h}$ of infection, and the efficiency of transfection was evaluated by qRT-PCR and Western blot assays.

\section{Western blot analysis and antibodies}

Protein was extracted from tissues and cells for protein analysis. The samples were boiled at $100^{\circ} \mathrm{C}$ for 5 min. Equal amounts of protein were separated by SDS-PAGE and transferred to PVDF membranes (Millipore, USA). The membranes were incubated with primary antibodies overnight at $4^{\circ} \mathrm{C}$. The immunoreactive protein bands were detected using an ECL detection system (Cell Signaling Technology, USA).

\section{Quantitative real-time polymerase chain reaction (qRT-PCR)}

The mRNA expression was quantified by real-time RT-PCR. Briefly, total RNA was extracted from the cell lines using TRIzol reagent (Ruisai Biotechnology Co., Ltd., Shanghai). After quantification, a total of $10 \mathrm{ng}$ RNA was used for CDNA synthesis, followed by real-time PCR using the One Step SYBR® PrimeScript ${ }^{\mathrm{TM}}$ RT-PCR Kit II (Ruisai Biotechnology Co., Ltd., Shanghai) according to the manufacturer's instructions. ACTB was employed as an internal control. The sequences of the primers used were as follows: TRIM44forward: 5'-GGCACTCTTCCAGCCTTCC-3'; TRIM44-reverse: 5'-GAGCCGCCGATCCACAC-3'; ACTB-forward: 5'-GCTGGTAACTTGTTGTGCGG-3'; ACTB-reverse: 5'-GAGCCGCCGATCCACAC-3'. PCR was carried out for 40 cycles $\left(95^{\circ} \mathrm{C}\right.$ for $15 \mathrm{~s}, 60^{\circ} \mathrm{C}$ for $20 \mathrm{~s}$ and $72^{\circ} \mathrm{C}$ for $20 \mathrm{~s}$ ) according to the instructions. Relative quantification of TRIM44 mRNA was conducted using the $2^{-\Delta \Delta C T}$ method, followed by normalization using the internal control.

\section{Cell counting kit-8 (CCK-8) assay}

ACCK-8 assay (CK04, Dojindo Molecular Technologies, Inc., Kumamoto, Japan) was performed to test cell proliferation. After being placed in a 96-well plate at an initial density of $5 \times 10^{3}$ cells/well, the SKOV3 and A2780 cells were transfected with shTRIM44 or shNC (lentivirus-infected cells). At each time point, cells in each well were incubated with $10 \mu \mathrm{l}$ of CCK-8 dye in $90 \mu \mathrm{l}$ of culture medium for $2 \mathrm{~h}$ at $37^{\circ} \mathrm{C}$. Then, the OD values at $450 \mathrm{~nm}$ were measured using a VERS Amax Microplate Reader (Molecular Devices Corp, Sunnyvale, CA).

\section{Flow cytometric analysis of apoptosis}


SKOV3 and A2780 cells were transfected with TRIM44-shRNA for $48 \mathrm{~h}$. The cells were harvested by centrifugation at $10,000 \times \mathrm{g}$ for $5 \mathrm{~min}$. The cells were washed twice with cold PBS, centrifuged at 10,000 $\times \mathrm{g}$ for $5 \mathrm{~min}$, and then resuspended in PBS. The cells were resuspended and stained with propidium iodide (PI, 1:100 dilution) and Annexin V-APC (Cell Signaling Technology, Danvers, U.S.A.) at $20^{\circ} \mathrm{C}$ for 30 min in the dark. The stained cells were analyzed using a BD FACSAria Cell Sorter.

\section{Migration and invasion assays}

The invasion and migration assays were assessed by Transwell filters (Corning Incorporated, Corning, NY, USA) with (invasion assay) or without Matrigel (migration assay). For the invasion assay, the Transwell chambers were pretreated with Matrigel (BD Bioscience, Mountain View, CA, USA). First, ovarian cell transfections were suspended in medium without FBS and seeded into the upper chamber. The lower chamber contained RPMI- 1640 with $10 \%$ FBS. After cultivation for $48 \mathrm{~h}$ at $37^{\circ} \mathrm{C}$, noninvasive cells on the upper chambers were removed by cotton swabs, while the invasive cells on the lower chamber were subsequently fixed and stained with $0.1 \%$ crystal violet for $15 \mathrm{~min}$. An inverted microscope (Olympus Corporation, Tokyo, Japan) was used to evaluate the invasion and migration.

\section{Colony formation assay}

Following lentivirus infection, SKOV3 and A2780 cells were plated in 6-well plates at a density of $1.5 \times 10^{3}$ cells/well. Then, the cells were cultured in a humidified incubator at $37^{\circ} \mathrm{C}$ with $5 \% \mathrm{CO}_{2}$. After 7 days, the natural colonies formed, and the cells were washed three times with PBS, fixed in $1.5 \mathrm{ml}$ of $4 \%$ paraformaldehyde and stained with crystal violet (Sigma-Aldrich) for $20 \mathrm{~min}$. After the samples were washed with PBS twice, the staining solution was removed, and the cells were air dried. Colony formation was observed through a light/fluorescence microscope to obtain the colony numbers.

\section{Nude mouse xenograft assays in vivo}

The animal study procedure was approved by the Animal Experimentation Ethics Committee of Harbin Medical University Cancer Hospital. Female athymic BALB/c nude mice (aged six weeks, weighing 20-22 g) were obtained from SLALaboratory Animal Co., Ltd. (Shanghai, China). The mice were housed in a pathogen-free animal facility and randomly assigned to the control or experimental group (six mice per group). The tumor volume was estimated every three days by the following formula: $0.5 \times$ length $\times$ width $^{2}$. All mice were sacrificed after 23 days. The tumor sizes were measured at least once or twice a week to monitor tumor growth.

\section{Statistical analysis}

Data are expressed as the mean \pm SD. Statistical analysis was performed with GraphPad Prism 7.0 software. The differences among groups were analyzed by ANOVA or Student's $t$ test. Data with $P<0.05$ were considered statistically significant. 


\section{Results}

\section{Increased expression of TRIM44 in the EOC tissues and cell lines}

We first examined the mRNA and protein levels of TRIM44 in 5 fresh EOC samples and 3 normal ovarian tissue samples. The results showed that the mRNA and protein expression of TRIM44 was significantly upregulated in the EOC samples compared to the normal noncancerous tissue samples (Figure 1A, B and C; $P<0.05)$. We then determined the TRIM44 expression in five human EOC cell lines: CAOV3, OVCAR3, A2780, HO-8910PM and SKOV3. Western blot analyses revealed that TRIM44 expression was the highest in the A2780 cells (Figure 1D and E; $P<0.05$ ). Taken together, these data suggest that TRIM44 may serve as a vital biomarker of EOC.

\section{Lentivirus-mediated RNAi significantly inhibited TRIM44 expression in SKOV3 and A2780 cells}

The individual shRNA oligos targeting TRIM44 were validated. The Western blot results indicated that lentiviral vectors with the TRIM44 shRNA sequence can effectively decrease the expression of TRIM44 in SKOV3 and A2780 cells (Figure $2 A$ and B).

\section{Knockdown of TRIM44 suppressed the SKOV3 and A2780 cell proliferation in vitro}

To detect whether TRIM44 influenced the malignant properties of EOC cells, we first performed cell proliferation analysis by CCK-8 assays. We found that knockdown of TRIM44 resulted in a significant reduction in cell proliferation (Figure $2 \mathrm{C}$ and $\mathrm{D}$ ). As shown in Figure 2, the growth curve of the shTRIM44treated cells started to drop from the third day compared with that of the nontreated and shNC-treated cells. The decline reached $54.14 \%$ and $76.93 \%$ for the shTRIM44-treated SKOV3 and A2780 cells, respectively, on the fifth day compared with the shNC-treated cells $(P<0.001)$.

\section{Knockdown of TRIM44 suppressed the invasion and migration of the SKOV3 and A2780 cells in vitro}

In the Transwell invasion assay, we observed that invasion was inhibited in the lenti-shTRIM44transfected cells. The migration experiment showed that knockdown of TRIM44 reduced the migration of the lenti-shTRIM44 virus-transfected cells (Figure 2E, F and H). The migration of the SKOV3 cells transfected with TRIM44 shRNA was significantly inhibited compared with that of the control cells $(0.115$ \pm 0.002 versus $0.258 \pm 0.002, P<0.001$ ). The migration of the A2780 cells transfected with TRIM44 shRNA was significantly inhibited $(0.030 \pm 0.003$ versus $0.086 \pm 0.006, P<0.001)$. The invasion experiment showed that knockdown of TRIM44 reduced the invasion of the lenti-shTRIM44 virustransfected cells (Figure 2E, F and G). The invasion of the SKOV3 cells transfected with TRIM44 shRNA was significantly inhibited compared with that of the control cells $(0.100 \pm 0.004$ versus $0.289 \pm 0.005, P<$ 0.001). The invasion of the A2780 cells transfected with TRIM44 shRNA was significantly inhibited ( $0.308 \pm 0.007$ versus $0.497 \pm 0.004, P<0.001)$. The above results indicate that lentiviral vectors with the TRIM44 shRNA sequence can effectively inhibit the migration and invasion induced by TRIM44. 
We investigated potential cellular apoptosis events during cell death caused by TRIM44 knockdown in the ovarian cancer cell lines SKOV3 and A2780. The results showed that apoptosis increased in both the SKOV3 and A2780 cell lines after transfection with lenti-shTRIM44 (Figure 2I and J). Depletion of TRIM44 caused drastic morphological changes and a substantial decrease in cell number. Taken together, these results clearly indicated that TRIM44 depletion led to apoptotic cell death in the SKOV3 and A2780 cancer cells.

\section{Knockdown of TRIM44 significantly inhibited the colony-forming ability of SKOV3 and A2780 cells}

The long-term effect of TRIM44 silencing on the colony-forming ability of SKOV3 and A2780 cells was determined by colony formation assays. The size of the independent colonies was much smaller in the shTRIM44-treated cells than in the nontreated and shNC-treated cells. Moreover, the number of colonies formed by the SKOV3 and A2780 cells was significantly $(P<0.05)$ decreased following TRIM44 knockdown (Figure $2 \mathrm{~K}$ and $\mathrm{L}$ ). The data indicated that TRIM44 knockdown also significantly inhibited colony formation in the SKOV3 and A2780 ovarian cancer cells.

\section{Knockdown of TRIM44 inhibited xenotransplanted tumor growth in nude mice in vivo}

To evaluate the effect of TRIM44 on ovarian carcinogenesis in vivo, we established xenograft tumor models in nude mice with A2780 cells transfected with lenti-shTRIM44 treatment. Xenograft tumors were observed in the nude mice at the injection site, and the tumors were harvested 30 days after injection. The tumor volumes were measured once a week. As shown in Figure3, the tumor volume in the lentishTRIM44-transfected cell treatment group was significantly decreased compared to that in the control group (Figure $4, P<0.05$ ). The above results indicated that lentiviral vectors with the TRIM44 shRNA sequence can inhibit xenotransplanted tumor growth, indicating that downregulation of TRIM44 may be beneficial for the treatment of ovarian cancer.

\section{Global gene expression analysis following TRIM44 knockdown revealed modulation of key pathways in epithelial ovarian cancer cells}

To elucidate the molecular mechanisms by which TRIM44 contributes to the carcinogenesis of lung cancer cells, we carried out gene chip analysis (gene expression profiling, using the Human Gene Expression Array PathArray ${ }^{\mathrm{TM}}$ platform) comparing the gene expression of SKOV3 NS cells versus SKOV3 shTRIM44 cells. Differentially expressed genes with at least a 1.2-fold change were identified. The common differentially expressed genes were analyzed by Ingenuity Pathway Analysis (IPA).We identified 1172 genes with significant expression changes ( $>1.2$-fold with adjusted $P<0.05$ ) (data not shown in this paper). The bioinformatics analysis showed that the expression of 436 genes was significantly increased $(P<0.05)$ and that of 736 genes was significantly decreased $(P<0.05)$. The most markedly altered genes were AKT1S1 (AKT1 substrate 1, decreased1.96-fold), USP14 (ubiquitin specific peptidase 14, decreased1.85-fold), STK38L (serine/threonine kinase 38 like, decreased1.83-fold), GLIPR1 (GLI pathogenesis-related 1, increased1.84-fold), SEC23A (Sec23 homolog A, COPII coat complex component, decreased 1.76-fold), and SPC24 (NDC80 kinetochore complex component, decreased1.72-fold). Many of 
these TRIM44-regulated differentially expressed genes in EOC cells are involved in regulating cell proliferation, wound healing, the cell cycle, DNA damage, and cellular assembly and organization. In addition, functional analysis of the genes using IPA revealed that differentially expressed genes were mainly enriched in the superpathway of cholesterol biosynthesis (ACAT2, FDPS, IDI1, LBR, LSS and MVD, etc.), the superpathway of serine and glycine biosynthesis I (PHGDH, PSAT1, PSPH, and SHMT2), cyclins and cell cycle regulation (CCNA1, CCNA2, CCNB2, CCND3, CCNE2, CDK1, E2F2,E2F8, etc.), estrogenmediated S-phase entry (CCNA1, CCNA2, CCNE2, CDK1,E2F2, etc.) the superpathway of geranylger anyldiphosphate biosynthesis I signaling (ACAT2, FDPS, IDI1, MVD, and MVK), the NER pathway (ACAT2, FDPS, IDI1, MVD, and MVK), cell cycle control of chromosomal replication (CDC6, CDK1, CDK10, LIG1, and MCM3,etc.), and oxidative phosphorylation (COX7A1, NDUFAB1, and NDUFB11, etc.). Taken together, these data indicate that TRIM44 may be a key regulator of cell proliferation and migration, therefore controlling cancer progression. Most of these pathways are implicated in tumorigenesis or chemotherapy resistance (all $P<0.05$; Figure 5).

\section{TRIM44 knockdown suppressed the FOXM1-EZH2 signaling pathway in epithelial ovarian cancer cells}

The potential gene network interacting with TRIM44 was also analyzed by IPA and showed that FOXM1EZH2 signaling was the downstream signaling pathway regulated by TRIM44 (Figure6). Subsequent Western blot assays showed that silencing TRIM44 dramatically downregulated the expression of several key proteins in the FOXM1-EZH2 signaling pathway, including FOXM1, EZH2, CCNE2, CCND3 and BIRC5 (Figure 6, $P<0.05$ ). These data further suggest that TRIM44 promotes human epithelial ovarian cancer progression via the FOXM1-EZH2 signaling pathway.

\section{Discussion}

In this study, we propose that TRIM44 acts as a metastasis-associated marker in the context of epithelial ovarian cancer (EOC) cells. Knockdown of TRIM44 inhibited the invasion of EOC cells, whereas ectopic expression of TRIM44 induced this activity. Gene chip analysis further showed that FOXM1-EZH2 signaling is involved in the epithelial ovarian cancer cell invasion and metastasis induced by TRIM44. Overexpression and knockdown of TRIM44 further confirmed that TRIM44 regulates invasion/metastasis by modulating the FOXM1-EZH2 signaling pathway.

The tripartite motif (TRIM) family is composed of genes that encode proteins containing TRIM. The integrated module comprises three different types of domains: a RING domain (R), a B-box domain (B), and a coiled-coil (CC) region (RBCC). The TRIM protein family is involved in a wide range of biological processes, such as cell growth, development, and cellular differentiation[13, 14]. Increasing evidence has shown that many TRIM family members are involved in the oncogenesis and progression of ovarian cancer. For instance, TRIM52 plays an oncogenic role in the development of ovarian cancer (OC)associated with the NF-kB signaling pathway[15]. Furthermore, it was demonstrated that knockdown of TRIM11 not only affects the expression of cell apoptosis-related and invasion-related proteins but also reduces the phosphorylation levels of ERK and AKT in ovarian cancer cells[16]. In addition, TRIM16 
inhibits migration and invasion by suppressing the Sonic hedgehog signaling pathway in ovarian cancer cells[17]. Given the correlation between the expression levels of these TRIMs and the tumorigenesis of OC, TRIMs represent potential novel therapeutic targets in patients with OC.

TRIM44 is one of the members of the tripartite motif (TRIM) family, which has been demonstrated to have a multifaceted role in cancer progression. Multiple reports have confirmed that TRIM44 is overexpressed in several human cancers and may promote tumor growth, proliferation and invasion[18]. Kashimoto $\mathrm{K}$ et al. showed that knockdown of TRIM44 expression inhibited the proliferation, migration, and invasion of TRIM44-overexpressing gastric cancer cells[10]. Another study identified and validated that TRIM44 is independently associated with poor outcome and provides additional prognostic information in esophageal adenocarcinoma[19]. Yamada $Y$ et al. also found that TRIM44 promotes cell proliferation and migration and inhibits apoptosis in testicular germ cell tumors[8].Our previous study found that high TRIM44 protein expression was strongly correlated with advanced histological grade, high FIGO stage and lymph node metastasis in patients with EOC[12]. Consistent with our previous data, the results of the present study indicated that TRIM44 was significantly expressed in OC tissue samples and cell lines. Furthermore, to explore the role of TRIM44 in ovarian cancer tumorigenesis, we knocked down TRIM44 in SKOV3 and A2780 cells. We successfully constructed a shRNA lentiviral vector that targeted TRIM44 and efficiently silenced the TRIM44 gene. In the present study, we showed for the first time that depletion of TRIM44 in SKOV3 and A2780 cells suppressed invasion, migration, proliferation, and colony formation while inducing cell apoptosis. Metabolically, knockdown of TRIM44 significantly inhibited tumor growth in vivo. Thus, our study is consistent with the finding that TRIM44 is essential formalignant transformation of tumor cells. These findings suggest that TRIM44 may act as an oncogene in EOC.

It has been reported that TRIM44 is involved in cancer development and progression by various mechanisms, such as promoting TNFa-dependent phosphorylation of the p65 subunit of NF-KB and IKBa in breast cancer cells[6], inducing cell EMT via MAPK signaling in intrahepatic cholangiocarcinoma or activating the AKT/mTOR signal transduction pathway in human esophageal cancer[19]. A study in human esophageal cancer demonstrated that TRIM44 can promote quiescent multiple myeloma cell occupancy and survival in the osteoblastic niche via HIF-1a stabilization[20]. In addition, a recent study found that knockdown of TRIM44 inhibits the proliferation and invasion of papillary thyroid cancer cells by suppressing the Wnt/ $\beta$-catenin signaling pathway. However, the underlying mechanisms of TRIM44 in ovarian cancer cell invasion and metastasis remain to be fully elucidated. In our study, gene chip data were analyzed by IPA. Gene expression profiling using the Affymetrix Human Gene 1.0 ST platform identified 1172 transcripts that were significantly differentially expressed, based on a $P<0.05$ threshold, in SKOV3 epithelial ovarian cancer cells with TRIM44 knockdown compared with the control cells. Functional analysis of the genes using IPA revealed that TRIM44 knockdown modulated key pathways typically activated in cancer.

Moreover, the potential gene network interacting with TRIM44 was analyzed by IPA and showed that FOXM1-EZH2 signaling was the downstream pathway regulated by TRIM44. The FOXM1-EZH2 signaling 
pathway is a well-characterized signaling pathway during the development of various cancer types[2123]. However, it is still unknown whether the key proteins of the FOXM1-EZH2 signaling pathway are functionally different or functionally redundant during the carcinogenic mechanism of TRIM44.

Subsequent Western blot experiments confirmed that FOXM1-EZH2 signaling was repressed by TRIM44 knockdown, suggesting that TRIM44 positively regulates the carcinogenesis of ovarian cancer cells through FOXM1-EZH2 signaling.

In conclusion, we showed reduced expression of TRIM44 transcript expression in human ovarian tumor tissues relative to normal ovarian tissues and inhibition of human epithelial ovarian cancer cell invasion, migration, and growth by TRIM44, at least inpart through suppression of FOXM1-EZH2 signaling. Our findings identify a potential role of the TRIM44 gene in human EOC pathogenesis by activating the FOXM1-EZH2 signaling pathway. These conclusions need to be further validated in larger experiments.

\section{Declarations}

\section{Acknowledgments}

We than Dr. Huike Yang for participating in the evaluation procedures.

\section{Authors' contributions}

Fanling Meng designed the study and statistical analysis and drafted the manuscript. Fanling Meng, Rong $\mathrm{Ma}$ and Ning Huang participated in the design of the study. Jing Ding, Lin Sui, Xihai Chen, Yuanlong Hu, Shuang Liu, Guangyao Shi, Yunlong He, Xin, Ning, Jiaqi Zhu, Ruixue Zhao, Jing Ma, Sujing Ren and Ning Huang collected the data and contributed to the composition and aim of this research. All authors read and approved the final manuscript.

\section{Ethics approval and consent to participate}

This study was approved by the Ethical Committee of Harbin Medical University Cancer Hospital.

\section{Consent for publication}

We obtained consent to publish the paper.

\section{Competing interests}

The authors declare that they have no competing interests.

\section{Availability of data and materials}

The data used and analyzed during the current study are available from the corresponding author on reasonable request.

\section{Funding}


This work was supported by grants of the Liande Wu Science Foundation for Young Scholars of Harbin Medical University Cancer Hospital (WLD-QN1705) and the Jingying Foundation of the Harbin Medical University Cancer Hospital (JY2015-04). The founders had no role in study design, data collection and analysis, decision to publish, or preparation of the manuscript.

\section{References}

1. Ferlay J, Soerjomataram I, Dikshit R, Eser S, Mathers C, Rebelo M, Parkin DM, Forman D, Bray F: Cancer incidence and mortality worldwide: sources, methods and major patterns in GLOBOCAN 2012. Int J Cancer 2015, 136(5):E359-386.

2. Siegel RL, Miller KD, Jemal A: Cancer statistics, 2015. CA Cancer J Clin 2015, 65(1):5-29.

3. Siegel RL, Miller KD, Jemal A: Cancer Statistics, 2017. CA Cancer J Clin 2017, 67(1):7-30.

4. Wang H, Fang ZL, Zhang GH, Ma X: TRIM44, a crucial target of miR-410, functions as a potential oncogene in osteosarcoma. Onco Targets Ther 2018, 11:3637-3647.

5. Luo Q, Lin H, Ye X, Huang J, Lu S, Xu L: Trim44 facilitates the migration and invasion of human lung cancer cells via the NF-kappaB signaling pathway. Int J Clin Oncol 2015, 20(3):508-517.

6. Kawabata H, Azuma K, Ikeda K, Sugitani I, Kinowaki K, Fujii T, Osaki A, Saeki T, Horie-Inoue K, Inoue S: TRIM44 Is a Poor Prognostic Factor for Breast Cancer Patients as a Modulator of NF-kappaB Signaling. Int J Mol Sci 2017, 18(9).

7. Zhou Z, Liu Y, Ma M, Chang L: Knockdown of TRIM44 inhibits the proliferation and invasion in papillary thyroid cancer cells through suppressing the Wnt/beta-catenin signaling pathway. Biomed Pharmacother 2017, 96:98-103.

8. Yamada Y, Takayama KI, Fujimura T, Ashikari D, Obinata D, Takahashi S, Ikeda K, Kakutani S, Urano T, Fukuhara $\mathrm{H}$ et al: A novel prognostic factor TRIM44 promotes cell proliferation and migration, and inhibits apoptosis in testicular germ cell tumor. Cancer Sci 2017, 108(1):32-41.

9. Tan Y, Yao H, Hu J, Liu L: Knockdown of TRIM44 Inhibits the Proliferation and Invasion in Prostate Cancer Cells. Oncol Res 2017, 25(8):1253-1259.

10. Kashimoto K, Komatsu S, Ichikawa D, Arita T, Konishi H, Nagata H, Takeshita H, Nishimura Y, Hirajima S, Kawaguchi T et al: Overexpression of TRIM44 contributes to malignant outcome in gastric carcinoma. Cancer Sci 2012, 103(11):2021-2026.

11. Ong CA, Shannon NB, Ross-Innes CS, O'Donovan M, Rueda OM, Hu DE, Kettunen MI, Walker CE, Noorani A, Hardwick RH et al: Amplification of TRIM44: pairing a prognostic target with potential therapeutic strategy. J Natl Cancer Inst 2014, 106(5).

12. Liu S, Yin H, Ji H, Zhu J, Ma R: Overexpression of TRIM44 is an independent marker for predicting poor prognosis in epithelial ovarian cancer. Exp Ther Med 2018, 16(4):3034-3040.

13. Hatakeyama S: TRIM Family Proteins: Roles in Autophagy, Immunity, and Carcinogenesis. Trends Biochem Sci 2017, 42(4):297-311. 
14. Cambiaghi V, Giuliani V, Lombardi S, Marinelli C, Toffalorio F, Pelicci PG: TRIM proteins in cancer. Adv Exp Med Bio/ 2012, 770:77-91.

15. Yang W, Liu L, Li C, Luo N, Chen R, Li L, Yu F, Cheng Z: TRIM52 plays an oncogenic role in ovarian cancer associated with NF-kB pathway. Cell Death Dis 2018, 9(9):908.

16. Chen Y, Sun J, Ma J: Proliferation and invasion of ovarian cancer cells are suppressed by knockdown of TRIM11. Oncol Lett 2017, 14(2):2125-2130.

17. Tan H, Qi J, Chu G, Liu Z: Tripartite Motif 16 Inhibits the Migration and Invasion in Ovarian Cancer Cells. Oncol Res 2017, 25(4):551-558.

18. Peng R, Zhang PF, Zhang C, Huang XY, Ding YB, Deng B, Bai DS, Xu YP: Elevated TRIM44 promotes intrahepatic cholangiocarcinoma progression by inducing cell EMT via MAPK signaling. Cancer Med 2018, 7(3):796-808.

19. Xiong D, Jin C, Ye X, Qiu B, Jianjun X, Zhu S, Xiang L, Wu H, Yongbing W: TRIM44 promotes human esophageal cancer progression via the AKT/mTOR pathway. Cancer Sci 2018, 109(10):3080-3092.

20. Chen Z, Lin TC, Bi X, Lu G, Dawson BC, Miranda R, Medeiros LJ, McNiece I, McCarty N: TRIM44 promotes quiescent multiple myeloma cell occupancy and survival in the osteoblastic niche via HIF1alpha stabilization. Leukemia 2018.

21. Kim SH, Joshi K, Ezhilarasan R, Myers TR, Siu J, Gu C, Nakano-Okuno M, Taylor D, Minata M, Sulman EP et al: EZH2 protects glioma stem cells from radiation-induced cell death in a MELK/FOXM1dependent manner. Stem Cell Reports 2015, 4(2):226-238.

22. Harzer $K$, Yildiz Y: High beta-glucosidase (GBA) activity not attributable to GBA1 and GBA2 in live normal and enzyme-deficient fibroblasts may emphasise the role of additional GBAs. Biol Chem 2015, 396(11):1241-1246.

23. Perotti V, Baldassari P, Molla A, Nicolini G, Bersani I, Grazia G, Benigni F, Maurichi A, Santinami M, Anichini A et al: An actionable axis linking NFATc2 to EZH2 controls the EMT-like program of melanoma cells. Oncogene 2019, 38(22):4384-4396.

\section{Figures}




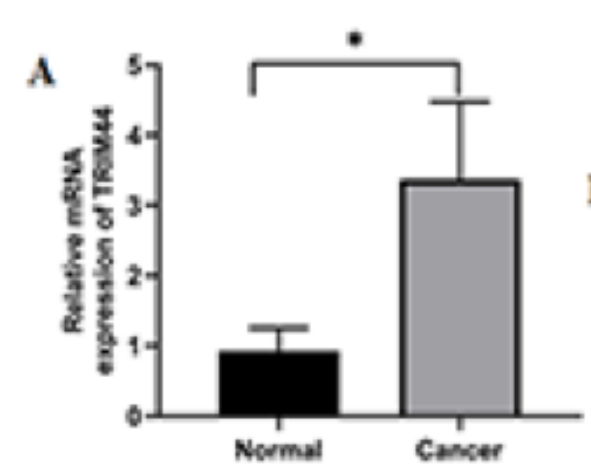

D

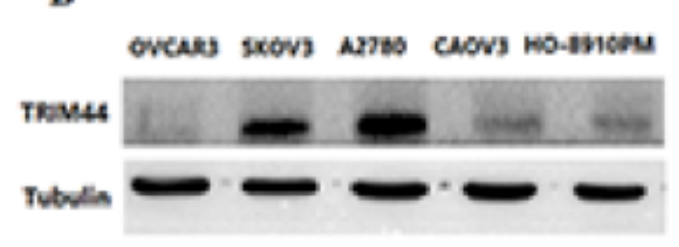

B
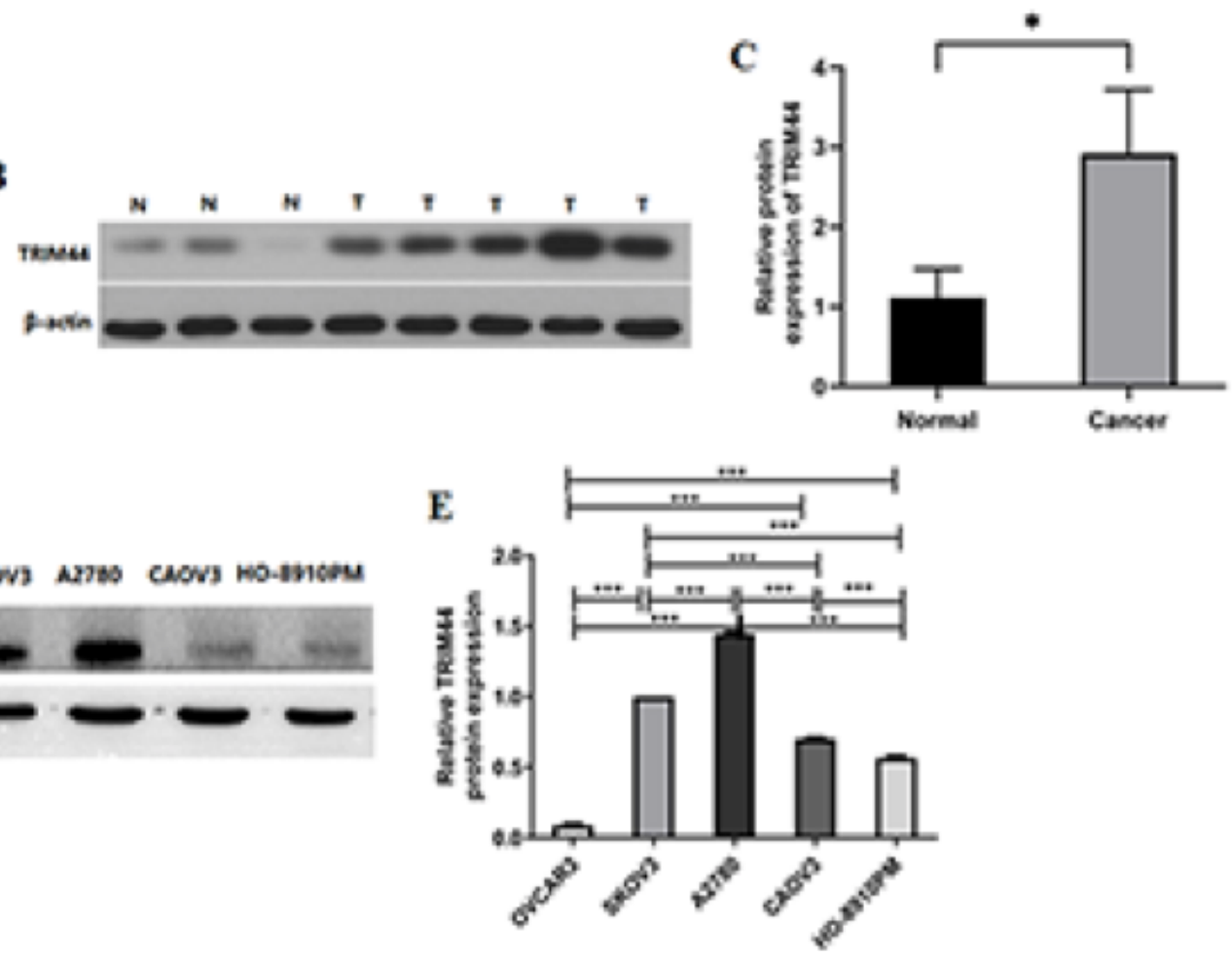

\section{Figure 1}

TRIM44expression is increased in epithelial ovarian cancer (EOC) cell lines and tissues. A, B and C, mRNA and protein expression of TRIM44 in 5 EOC tissue samples and 3 normal ovarian tissues.TRIM44 expression was elevated in the EOC samples compared with the $\mathrm{N}$ samples (data represent the mean \pm $\mathrm{SD}) ; \mathrm{D}$ and $\mathrm{E}$, the protein expression of TRIM44 was examined by Western blots in 5 EOC cell lines $(\mathrm{P}<$ 0.05). 

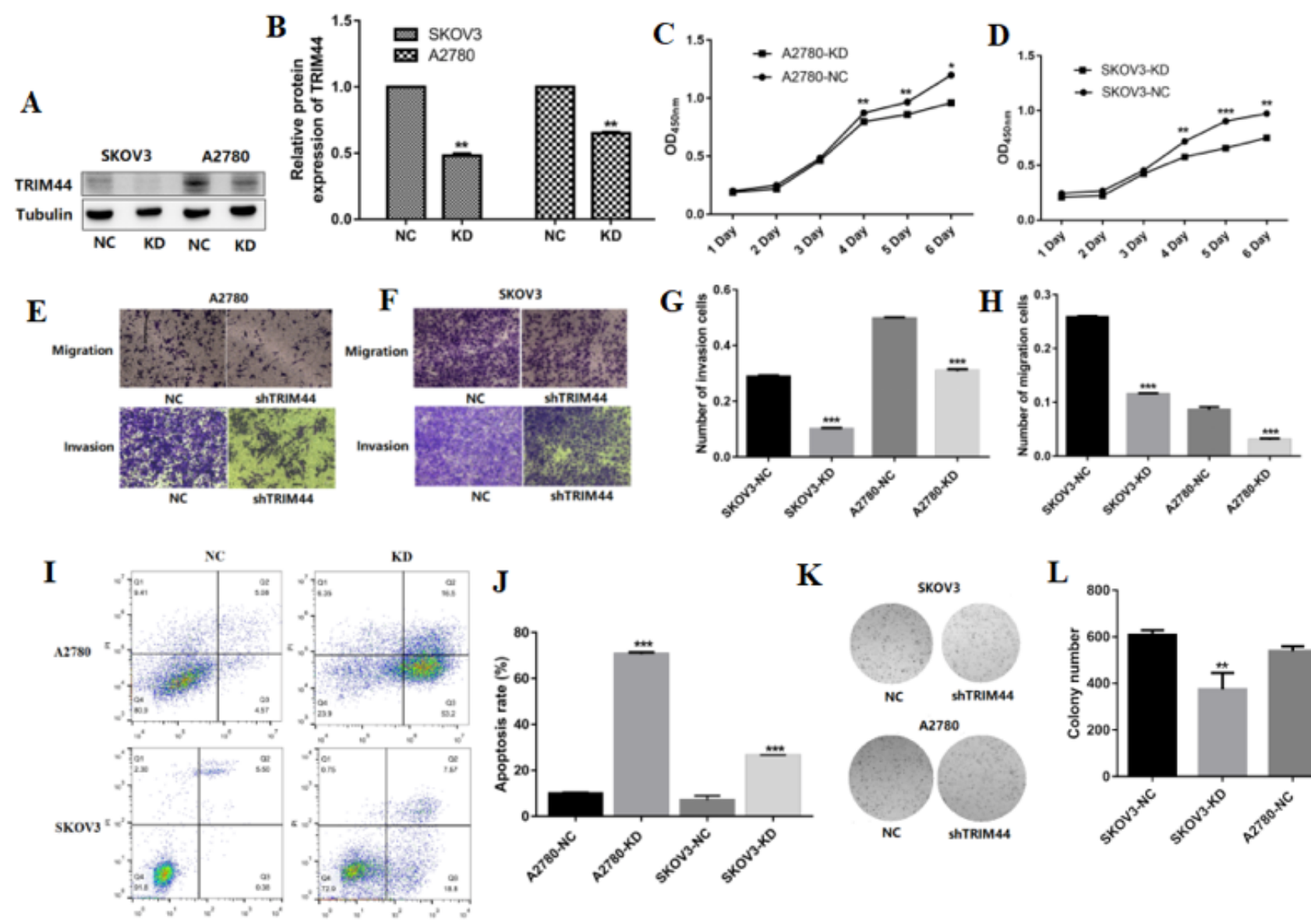

$\mathbf{K}$
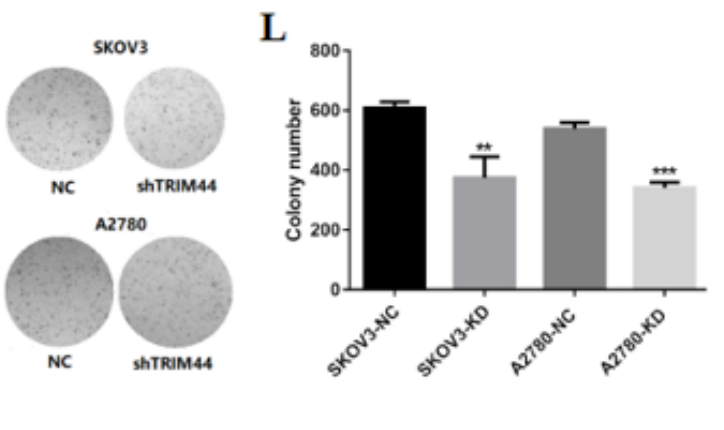

Figure 2

Effects of TRIM44 on the cell proliferation, migration, invasion, apoptosis and colony formation of EOC cells. A and B, SKOV3 and A2780 cells were infected with a TRIM44 shRNA-expressing lentivirus, and the expression of TRIM44 was examined by Western blots; $C$ and D, The effect of TRIM44 on cell viability was measured by the CCK-8 assays; E, F, G and H, Representative images and quantification of the Transwell migration and invasion assay data from the indicated cells; I and J, The effect of TRIM44 on cell apoptosis was measured by apoptosis assays; $\mathrm{K}$ and $\mathrm{L}$, Representative images and quantification of colony formation data from the shTRIM44- or TRIM44-infected EOC cells. ( ${ }^{\star} P<0.05$, ${ }^{\star \star} P<0.01$; data represent the mean \pm SD.) 


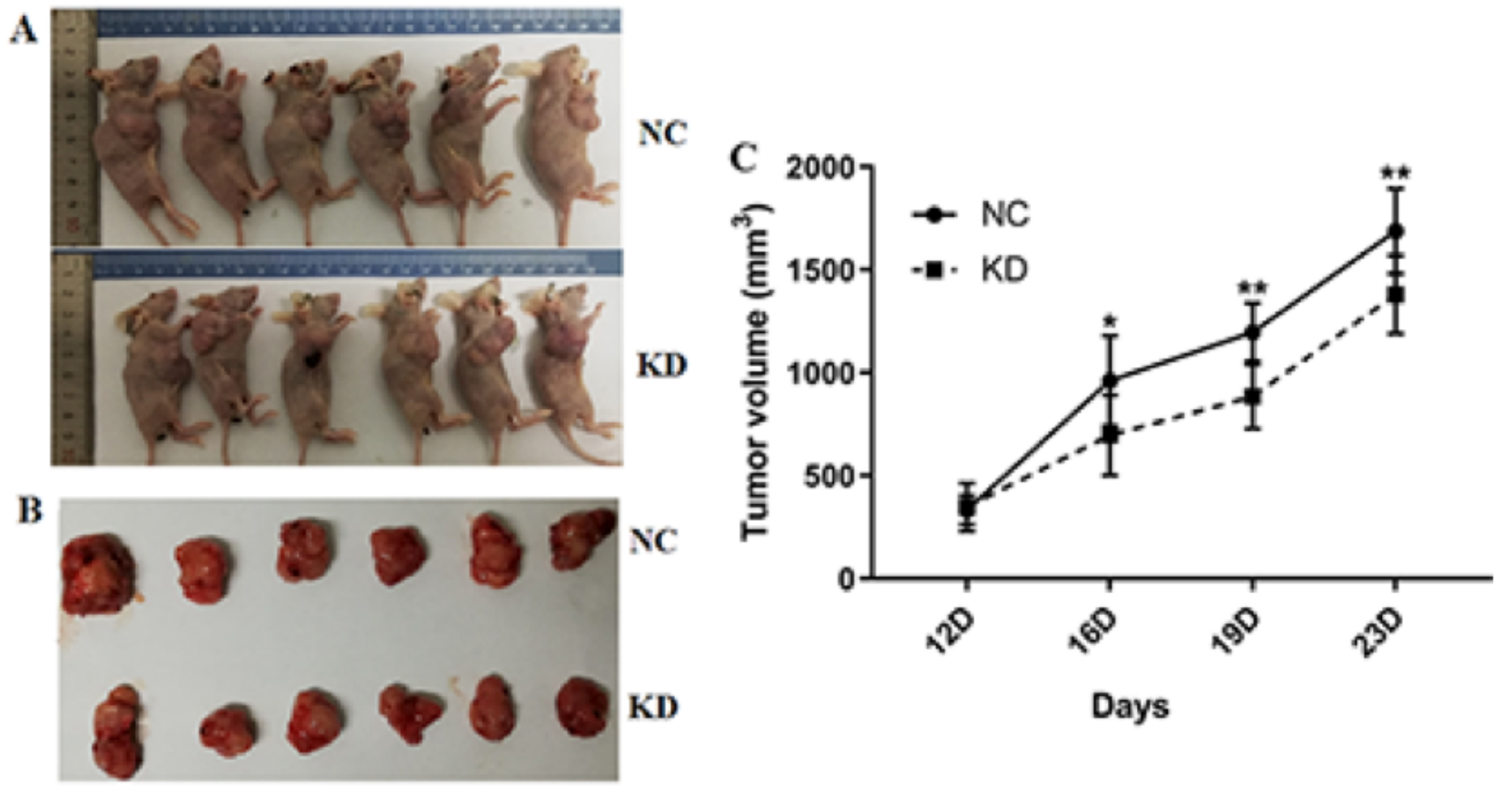

Figure 3

TRIM44 promotes epithelial ovarian cancer growth in vivo. A, SKOV3 cells expressing shTRIM44 were subcutaneously injected into nude mice, and the mice were monitored for $23 \mathrm{~d}$. B, A representative image of the tumors is shown; $C$, a growth curve of the tumor volumes was constructed. ${ }^{*} P<0.05,{ }^{*} P<0.01$. Data represent the mean \pm SD.

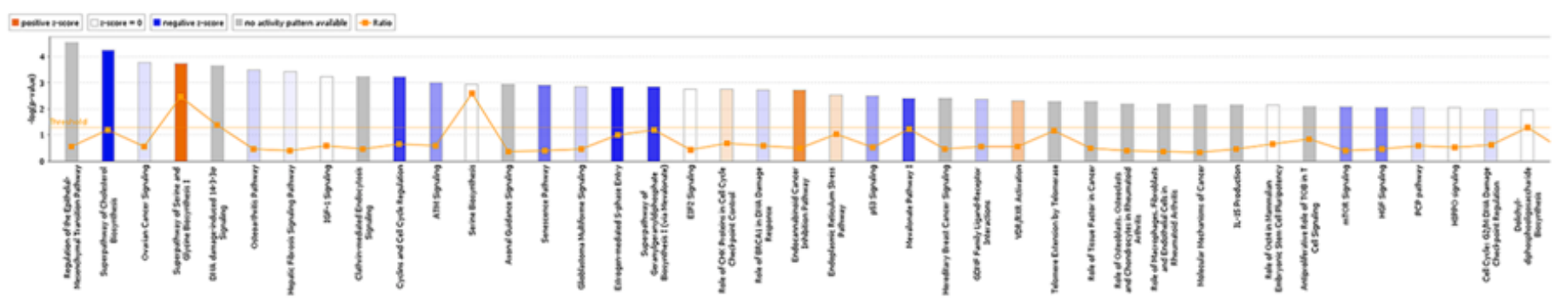

\section{Figure 4}

The canonical pathways for the differentially expressed genes in SKOV3 cells following TRIM44 knockdown. Several canonical pathways $(P<0.05)$ are shown in this figure. The line represents $P=0.05$. The ratio indicates the proportion of genes involved in some pathways among all the genes involved in this pathway. 


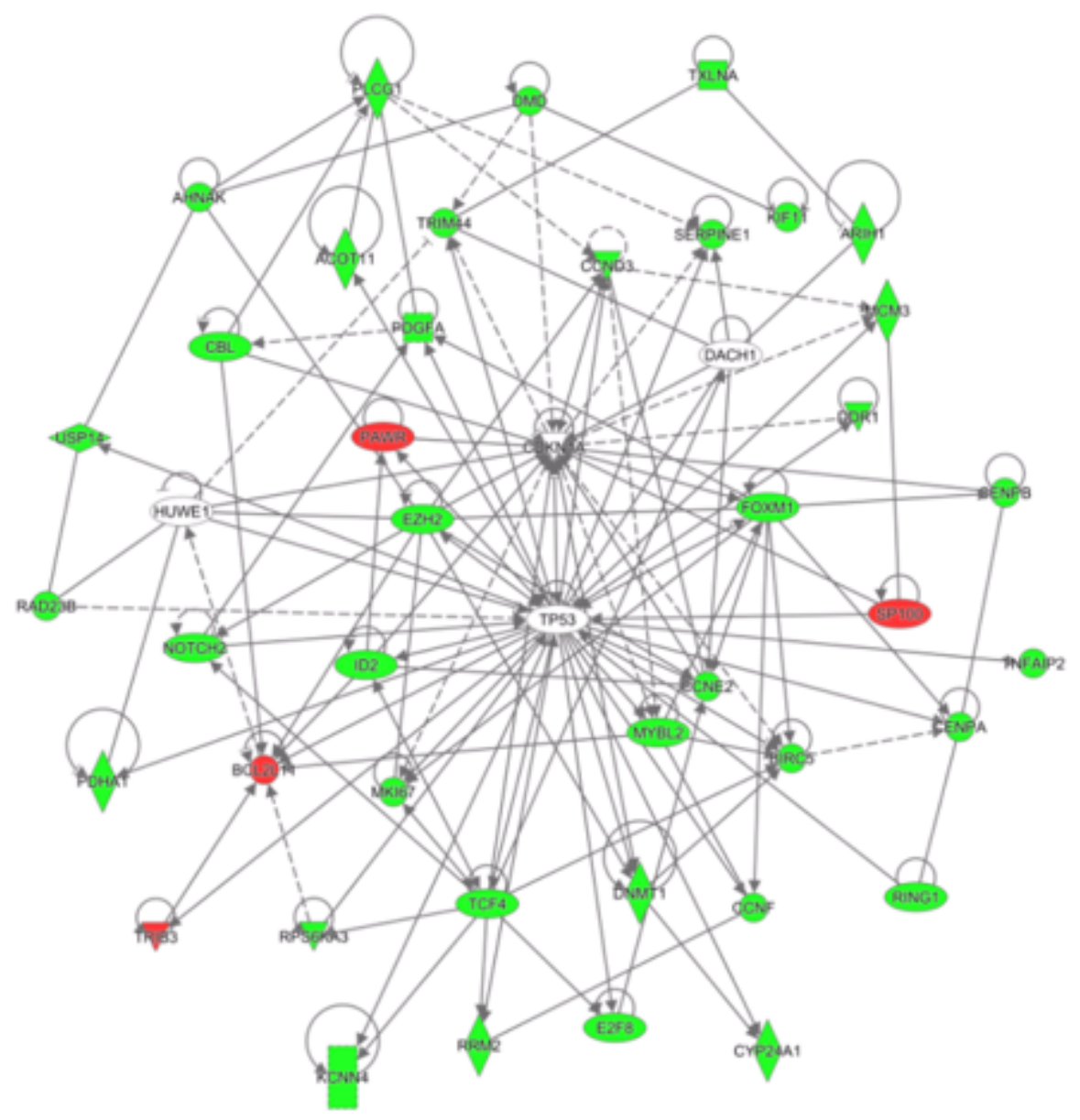

Figure 5

The potential gene network interacting with TRIM44 was also analyzed by IPA and found that FOXM1EZH2 signaling was the downstream pathway regulated by TRIM44. 




\section{Figure 6}

Western blotting of key proteins of the FOXM1-EZH2 pathway in the TRIM44-silenced SKOV3 cells. Proteins from cells treated with negative control (NC) shRNAs or TRIM44 were used to investigate the protein expression of FOXM1, EZH2, CCNE2, CCND3 and BIRC5. Total GAPDH was used as a loading control. 


\section{Supplementary Files}

This is a list of supplementary files associated with this preprint. Click to download.

- data.7z 